\title{
Prognostic Value of Neutrophil-Lymphocyte Ratio, D-Dimer Levels, and Scoring of Lung Lesion on Chest X-Ray on Mortality of COVID-19 Patients
}

\author{
(Which is the Best?)
}

\author{
Yudith Yunia Kusmala*, Jusron Iriawan \\ Internal Medicine Department, Faculty of Medicine \\ Jenderal Achmad Yani University \\ Cimahi, Indonesia \\ *yudith.yunia@lecture.unjani.ac.id
}

\author{
Fariz Albarri, Tersia Viradanti, Sunnatul Mufarrohah \\ General Medicine Study Program, Faculty of Medicine \\ Jenderal Achmad Yani University \\ Cimahi, Indonesia
}

\begin{abstract}
Acute Respiratory Distress Syndrome (ARDS) plays an important role that increases mortality in COVID-19 patients. Several parameters have been tried to predict the patient's condition for worsening and even death. The objective of this study was to determine the prognostic value of the neutrophil-lymphocyte ratio (NLR), D-Dimer levels, and the degree of severity of lung lesions on chest $\mathrm{X}$-rays on patient mortality. A cross-sectional restrospective study was conducted on 53 patients with positive PCR at Kebon Jati Hospital Bandung for the period September-December 2020. Each patient who was admitted, carried out routine examinations of NLR, D-Dimer, and chest $X$-ray. There were $40(75.5 \%)$ survival patients and 13 (24.5\%) patients died. The mean NLR, D-Dimer, and score of lung lesions on chest $\mathrm{X}$-rays of patients who died were higher than those of the survivor. NLR was 8.59 vs $4.10 ; P=0.002$, $D$ Dimer was $3,285.08$ vs $1,489.33 ; P=0.00$ and scoring for lung lesions on chest $X$-ray was 11.00 vs $6.82 ; P=0.001$. The predictive ability of these parameters shown by the Area Under Curve (AUC), with the highest predictive value was represented by the AUC NLR of $0.0840, P=0.000$. The cut-off NLR value was 5.07 with a sensitivity of $92.3 \%$ and a specificity of $79.5 \%$ in predicting the occurrence of death due to COVID-19. The results showed that the NLR, D-Dimer, and thoracic lesion scoring at the time of admission to the hospital have prognostic values for the mortality of COVID-19 patients.
\end{abstract}

Keywords-prognostic value, neutrophil-lymphocyte ratio, Ddimer, chest X-ray scoring, COVID-19

\section{INTRODUCTION}

Coronavirus Disease 19 (COVID-19) has been declared as world pandemic by WHO on January 30, 2020. COVID-19 is a zoonotic disease that is transmitted from animals to humans. It is known that this disease began in the city of Wuhan, China in December 2019. After that, the disease spread widely to 216 countries in various parts of the world [1].
Most patients with COVID-19 infection reportedly experience mild cold symptoms, including fever, cough, and fatigue. In patients with severe infection, the disease developed rapidly into acute respiratory syndrome (ARDS), coagulopathy, septic shock, and even death. The incidence rate of ARDS is around $19.6 \%$ and the incidence increases in non-survivor patients by $81 \%$, so this ARDS has a high role in the death of COVID-19 [2,3].

Post mortem study suggested that lung lesions in patients with COVID-19 are different from typical pre-COVID-19 ARDS, predominantly showed diffuse alveolar damage, formation of hyaline membranes, and the presence of platelet fibrin thrombi in the small arterial vessel is consistent with coagulopathy. They also found the inflammatory infiltrate composed of macrophages in the alveolar lumina [4]. Recent studies have shown that lymphopenia with cytokine storm syndrome is found frequently among patients with COVID-19. These features could reveal that the adjusted immune system plays a key role in determining disease progress [5]. The imaging feature appears bilateral lesions and lower lung predominant and multifocal. They often have the appearance of rounded opacities, with the development of ARDS the extend of lung involvement increases.

There were several routine assessments for each patient when they arrived at the hospital and were suspected as a COVID-19 patient. The parameters consist of hemoglobin, leucocyte and differential count, glucose level, C-reactive protein level, renal functions tests, liver function tests, and chest X-ray.

Recently we still couldn't find the studies comparing NLR neutrophil-lymphocyte ratio, D-dimer levels, and chest-Xrays, which is the best on predicting mortality in COVID-19 patients. D-dimer reflecting microthrombus formation, NLR reflecting dysregulation immune system, and lesions on the chest X-ray reflecting diffuse alveolar damage. 
The objective of this study was to determine the prognostic value of the neutrophil-lymphocyte ratio (NLR), D-dimer levels, and the degree of severity of lung lesions on chest $\mathrm{X}$ rays, which is the best on predicting mortality in COVID-19 patients.

\section{Methods}

This study was a restrospective observational single-center study that has been approved by the local Ethical Committee. A group of 53 patients with confirmed COVID-19 hospitalized in Kebon Jati Hospital Bandung from 1 Nov 2020 to 30 Jan 2021 were resrospectively analyzed. COVID-19 diagnostic was performed via molecular assay from nasopharyngeal swab. From each patient, neutrophil-lymphocyte ratio, D-dimer, and chest X-ray in admission were collected. Based on the clinical outcome, the patient group was divided into survivor and nonsurvivor groups. Peripheral blood samples were collected to examine NLR and D-Dimer. Quantification chest X-ray was made based on Brixia scoring. One radiologist, one internist, and one general practitioner were involved in assessing this quantification. The number of the score was the mean number of these three examiners [6]. Chest X-ray score $<4$ was mild, score 5-8 was moderate, score $>8$ was severe.

Descriptive analysis was presented as mean +SD for continuous variable and as percentages for categorical variable. Numerical data are statistically tested for significance using the two-sample t-test or the Mann-Witney test. The area under curve operating characteristic (ROC) was used to establish predictive ability. The cut-off point was obtained by determining the optimal den index (sensitivity+specificity-1). A p-value of less than 0,5 is considered significant. All analyses were performed using SPSS 24.0 version.

\section{RESUltS}

There were 29 male patients $(54,7 \%)$ and 24 female patients $(45,3 \%)$, with mean age was 52,39 years, the minimum year was 25 years old and the maximum age was Equations

TABLE I. THE FEATURE OF SEVERAL VARIABLE IN COVID-19 PATIENTS

\begin{tabular}{|l|l|l|}
\hline \multicolumn{1}{|c|}{ Variables } & \multicolumn{1}{c|}{ Mean \pm SD } & Percentage \\
\hline D dimer & $1938,27+2696,76$ & \\
\hline NLR & $5,22+4,63$ & \\
\hline Chest X-ray Scoring & $7,86+3,59$ & \\
\hline States & & $40(75,5 \%)$ \\
\hline Survival & $13(24,5 \%)$ \\
\hline Non-survival & \multicolumn{2}{|l}{} \\
\hline Chest X Ray interpretation & $11(20,8 \%)$ \\
\hline Mild & $15(28,3 \%)$ \\
\hline Moderate & $27(50,9 \%)$ \\
\hline Severe &
\end{tabular}

There were 40 patients who survived $(75,5 \%)$ and 13 patients died $(24,5 \%)$ and $50,9 \%$ had severe lesions in chest Xrays based on Brixia scoring (Table 1). Based on Table 2, D- dimer, NLR, and chest X-ray scoring had significant differences in mortality in COVID-19 patients.

TABLE II. ASSOCIATED D-DIMER, NLR, CHEST X-RAY AND MORTALITY

\begin{tabular}{|l|l|l|l|}
\hline \multicolumn{1}{|c|}{ Variable } & \multicolumn{1}{|c|}{$\begin{array}{c}\text { Survival } \\
\text { (mean + SD) }\end{array}$} & \multicolumn{1}{|c|}{$\begin{array}{c}\text { Non survival } \\
\text { (mean + SD) }\end{array}$} & P Value \\
\hline D-dimer & $1489,33+2116,90$ & $3285,08+3754,67$ & 0,000 \\
\hline NLR & $4,10+3,97$ & $8,59+4,96$ & 0,002 \\
\hline $\begin{array}{l}\text { Chest X-Ray } \\
\text { Scoring }\end{array}$ & $6,82+2,98$ & $11,00+3,53$ & 0,001 \\
\hline
\end{tabular}
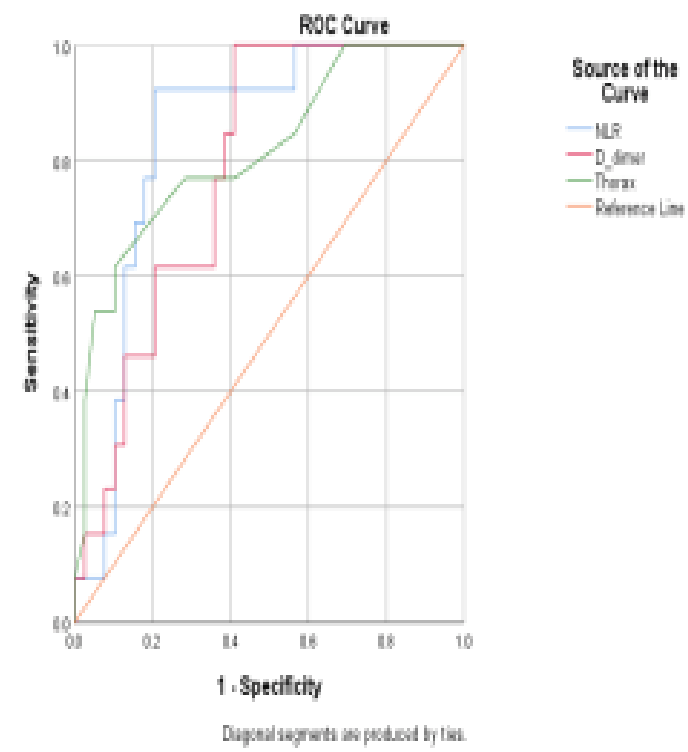

Fig. 1. Receiver operating characteristic curve for NLR, D-Dimer, and ches $\mathrm{X}$-ray as the parameter for predicting in hospital mortality in COVID-19 patients.

As the same result based on fig.1, we could find that NLR, $\mathrm{D}$-dimer, and chest $\mathrm{X}$-ray scoring have a good diagnostic value to predict mortality because their curve lies above the reference line (orange line). The higher area under the curve belongs to NLR (0,840 CI: 95\%), 93,2\% of sensitivity and 79,5\% of specificity with the cut-off value was 5,07 (Table 3)

TABLE III. TABle AREA Under the Curve (AUC) Histoscore

\begin{tabular}{|l|l|l|l|l|l|}
\hline Variable & $\begin{array}{c}\text { AUC } \\
(\mathbf{9 5 \%} \text { CI) }\end{array}$ & $\begin{array}{c}\text { Cut off } \\
\text { value }\end{array}$ & Sensitivity & Specificity & $\begin{array}{c}\text { P } \\
\text { value }\end{array}$ \\
\hline NLR & 0,840 & 5,07 & $92,3 \%$ & $79,5 \%$. & 0,000 \\
\hline D-Dimer & 0,785 & 1362 & $69,2 \%$ & $64,1 \%$ & 0,002 \\
\hline $\begin{array}{l}\text { Chest X- } \\
\text { ray }\end{array}$ & 0,816 & 8,5 & $76,9 \%$ & $71,8 \%$ & 0,001 \\
\hline
\end{tabular}

\section{DISCUSSION}

Early identification of risk factors for severe patients is very important to serve appropriate supportive care or access to ICU if necessary. Several studies have shown that severe COVID- 
19 patients have higher neutrophil levels and lower lymphocyte count. An elevated NLR could be a useful factor in the reflection of imbalance between inflammatory and immune response. The mechanism underlying this situation has yet to be determined. Lymphocytes play an important role in the regulation of immune response and decrease in the number of lymphocytes associated with non-resolution of inflammation [7]. A well-coordinated innate immune response is the first line of defense against viral infections. If there is dysregulation of this first defense, there will be excessive infiltration of inflammatory cells, a storm of inflammation and even death can occur. Previous research on SARS-COV and MERS-COV has shown that $\mathrm{T}$ cells, however, play an important role in preventing or controlling the overactive innate immune response during this viral infection. CD4+ $\mathrm{T}$ cells will coordinate to regulate the immune response. CD4+ $\mathrm{T}$ cells facilitate the production of virus-specific antibodies through the activation of $\mathrm{B}$ cells. Furthermore, CD8+ $\mathrm{T}$ cells mainly perform their tasks through 2 mechanisms, namely cytokine secretion and cytolytic activity against target cells. Secretion of cytokines such as secretion of IFN- $\gamma$ is very important to combat bacterial and viral infections. Recent studies have shown that $\mathrm{T}$ lymphocyte activity plays a more prominent role than B cells in regulating the pathogenesis and outcome of SARS-COV infections. In SARS-COV, as well as in severe COVID-19 infection, $\mathrm{T}$ lymphocytes decreased more. The mechanism behind progressive lymphopenia in severe COVID19 remains unclear. Possible mechanisms have been suggested including TNF- $\alpha$ mediated T cell apoptosis or direct cytopathic injury to T cell lymphocyte. COVID-19 will attack T cells and damage the immune system and cause more serious infections. It is also important to note that immune-cell infiltration can lead to excessive secretion of proteases and reactive oxygen species, fostering further damage and hyperinflammation. The pathological damage resulting from COVID-19 infection is strongly associated with pulmonary neutrophil infiltration and increased neutrophils in the peripheral circulation [8].

There is a correlation between chest X-ray pattern temporally change and severity of COVID-19. The chest X-ray result evolves from typical ground glass opacity to predominantly consolidative at the same time as the progression of the disease. So that it describes how far lung parenchyma involvement in the disease.

Previous study showed a positive correlation between Ddimer level and chest X-ray scores in COVID-19 patients. It means that the higher D-dimer level, the higher chest X-ray scores result. This demonstrates us how coagulopathy is related to the severity of lung parenchyma involvement in COVID-19 disease. This finding is similar to some previous research reporting the elevated D-dimer level has a relation with the severity of pneumonia caused by coagulation dysregulation due to excessive inflammatory mediators induced by SARS-CoV-2 infection. Moreover, proinflammatory cytokine may be involved in endothelial injury then activates coagulation cascade and inhibits fibrinolysis so that D-dimer is commonly used as a biomarker to see the deterioration of COVID-19 patients besides chest X-ray [9].

Elevated D-dimer is the one of abnormal laboratory parameters found in patients with COVID-19 infection. Ddimer is the principal breakdown fragment of fibrin and is used as a biomarker of fibrin formation and degradation. The fibrin degradation product released upon cleavage of cross-linked fibrin by plasmin. Numerous studies have shown that D-dimer is often utilized in diagnosing disseminated intravascular coagulation, and pretest probability for deep vein thrombosis and pulmonary embolism [10]

Nil Guler et al. states that the increased D-dimer in COVID-19 may be related to virus life cycle that induced apoptotic process in the host cell target. Viral adhesion to the ACE2 receptor, entering the host cell, and will be followed by viral replication, and try to keep the host cell alive because they use the organelles to produce structures of virus, and after finishing this process, they killed the host cell. During this process, the virus interferes the apoptotic pathway and induced the immune system to start to interact with this process. All of these processes will cause infiltration of inflammatory cells, apoptosis of endothelial cells, and microvascular thrombosis. The apoptotic processes target the endothelial cells of the vasculature resulting in triggered coagulopathy via complement activation pathways and increased D-dimer. Uncontrolled apoptosis of alveolar endothelial cells can induce thrombi in the alveolar vascular bed and thrombi will make hypoxic injury and vascular fluid leaks to the lung tissue. The increasing level of D-dimer is linked to the severity and mortality in COVID-19 [11].

In this study, we found that D-dimer level has the lowest grade to predict mortality. Several factors can interfere with the level of D-dimer such as pregnancy, cancer, chronic liver disease, chronic kidney disease, post-trauma and surgery status, and vasculitis are also considered comorbidities for COVID19.

\section{CONCLUSION}

NLR has a higher prognostic value to predict mortality in severe COVID-19 patients compare with D-dimer and chest Xray scoring. This suggests that this parameter may be used early as a marker severity among the others and clinical staging. These findings implicate that further study to decreased coagulopathy with anticoagulant and antiinflammatory drugs treatment in the severe COVID-19 patients has the possibility of preventing worsening of dysregulation immune response and coagulopathy, and will help the medical field to develop an effective clinical strategy.

\section{ACKNOWLEDGMENT}

This study supported by Lembaga Pengabdian kepada Masyarakat (LPPM) Universitas Jenderal Achmad Yani, Cimahi. West Java, Indonesia. 


\section{REFERENCES}

[1] A. Parasher, "COVID-19: Current Understanding of Its Pathophysiology, Clinical Presentation and Treatment," Postgrad. Med. J., vol. 97, no. 1147, pp. 312-320, 2021.

[2] M.K. Bohn, A. Hall, L. Sepiashvili, B. Jung, S. Steele, and K. Adeli, "Pathophysiology of COVID-19: Mechanisms Underlying Disease Severity and Progression," Physiology, vol. 35, no. 5, pp. 288-301, 2020.

[3] S.S. Hasan, T. Capstick, R. Ahmed, C.S. Kow, F. Mazhar, H.A. Merchant, and S.T.R. Zaidi, "Mortality in COVID-19 Patients with Acute Respiratory Distress Syndrome and Corticosteroids Use: A Systematic Review and Meta-Analysis,” Expert Rev. Respir. Med., vol. 14, no. 11, pp. 1149-1163, 2020.

[4] L. Carsana, A. Sonzogni, A. Nasr, R.S. Rossi, A. Pellegrinelli, P. Zerbi, R. Rech, R. Colombo, S. Antinori, and M. Corbellino, "Pulmonary PostMortem Findings in a Series of COVID-19 Cases from Northern Italy: A Two-Centre Descriptive Study,” Lancet Infect. Dis., vol. 20, no. 10, pp. $1135-1140,2020$

[5] A. Saghazadeh and N. Rezaei, "Immune-Epidemiological Parameters of the Novel Coronavirus-a Perspective," Expert Rev. Clin. Immunol., vol. 16, no. 5, pp. 465-470, 2020.

[6] A. Borghesi and R. Maroldi, "COVID-19 Outbreak in Italy: Experimental Chest X-Ray Scoring System for Quantifying and
Monitoring Disease Progression," Radiol. Med., vol. 125, no. 5, pp 509-513, 2020.

[7] H. Wang, Y. Zhang, P. Mo, J. Liu, H. Wang, F. Wang, and Q. Zhao, "Neutrophil to CD4+ Lymphocyte Ratio as a Potential Biomarker in Predicting Virus Negative Conversion Time in COVID-19," Int. Immunopharmacol., vol. 85, p. 106683, 2020.

[8] M. Kong, H. Zhang, X. Cao, X. Mao, and Z. Lu, "Higher Level of Neutrophil-to-Lymphocyte Is Associated with Severe COVID-19," Epidemiol. Infect., vol. 148, 2020.

[9] Y. Yao, J. Cao, Q. Wang, Q. Shi, K. Liu, Z. Luo, X. Chen, S. Chen, K. $\mathrm{Yu}$, and Z. Huang, "D-Dimer as a Biomarker for Disease Severity and Mortality in COVID-19 Patients: A Case Control Study," J. intensive care, vol. 8, no. 1, pp. 1-11, 2020.

[10] S. Shah, K. Shah, S.B. Patel, F.S. Patel, M. Osman, P. Velagapudi, M.K. Turagam, D. Lakkireddy, and J. Garg, "Elevated D-Dimer Levels Are Associated with Increased Risk of Mortality in Coronavirus Disease 2019: A Systematic Review and Meta-Analysis," Cardiol. Rev., 2020.

[11] N. Guler, F. Siddiqui, and J. Fareed, "Is the Reason of Increased DDimer Levels in COVID-19 Because of ACE-2-Induced Apoptosis in Endothelium?," Clin. Appl. Thromb., vol. 26, p. 1076029620935526 , 2020. 